\title{
DIFERENÇAS DE GÉNERO NA CONCILIAÇÃO PROFISSIONAL E FAMILIAR
}

\author{
Susana Pereira ${ }^{1}$ \\ Célia Ribeiro ${ }^{2}$ \\ Paulo Pereira ${ }^{3}$
}

\begin{abstract}
Resumo: Existe uma composição social, tanto do trabalho, como do género, sendo conceitos que em outro contexto podem assumir outros significados. $O$ trabalho e a família ao longo dos tempos não têm apenas assumido papéis separados mas também segregados segundo o género. Neste artigo faz-se referência às perspetivas do género na conciliação entre o domínio profissional e o domínio familiar, dando ênfase ao papel da mulher. As investigações efetuadas neste âmbito não são consensuais quanto aos resultados encontrados, pois se algumas demostram que as mulheres vivenciam mais pressão e stress ao participarem nos dois domínios, familiar e profissional, e deste modo percecionam maior conflito que os homens, outras investigações indicam uma ausência de diferenças de género, qualquer que seja o sentido. Assim, podemos considerar que o conflito entre o trabalho e a família não é um problema apenas da esfera masculina ou feminina, mas sim das estruturas atuais do trabalho e da família que dificultam a conciliação das responsabilidades profissionais e familiares para ambos os géneros.
\end{abstract}

Palavras-chave: Género, Trabalho, Família, Conflito, Conciliação.

\footnotetext{
${ }^{1}$ Mestre em Gestão, Especialização em Gestão de Recursos Humanos. Consultora e formadora na área de segurança, higiene e saúde no trabalho e recursos humanos. Email: scasp@sapo.pt

2 Doutorada em Psicologia, área de especialidade em Psicologia Pedagógica.. Professora Auxiliar da Universidade Católica Portuguesa - Viseu. E-mail: cribeiro@viseu.ucp.pt

3 Prof. Auxiliar - Departamento de Economia, Gestão e Ciências Sociais da Universidade Católica Portuguesa. E-mail: ppereira@ viseu.ucp.pt
} 


\title{
Title: GENDER DIFFERENCES BETWEEN PROFESSIONAL AND FAMILY CONCILIATION
}

\begin{abstract}
There is a social composition, both work and the gender, and are concepts that in another context can take on other meanings. Work and family over the years have not only taken separate roles but also segregated by gender. In this article reference is made to gender perspectives in reconciling the professional domain and the family domain, emphasizing the role of women. Investigations in this area are not consensual as to the findings, because if some demonstrate that women experience more pressure and stress by participating in the two areas, familiar and professional, and thus perceive greater conflict than men, other studies indicate an absence of gender differences, whatever the direction. So we can consider that the conflict between work and family is not just a problem of male or female spheres, but also of the current structures of work and family that hinder the reconciliation of professional and family responsibilities for both genders.
\end{abstract}

Keywords: Gender, Work, Family, Conflict, Conciliation.

\section{INTRODUÇÃO}

As famílias são cada vez mais complexas e marcadas pela diversidade (Perista, 2002; Santos \& Gonçalves, 2011). A entrada massiva das mulheres no mercado de trabalho constitui uma das profundas mudanças decorrente das últimas décadas, que originou uma progressiva alteração dos papéis do género, tanto no trabalho como na família (Torres, 2004), com importantes implicações na forma como homens e mulheres organizam e desempenham socialmente os seus papéis (Pimenta \& Ribeiro, 2014).

Assim, novos desafios e dilemas foram criados para as atuais gerações portuguesas devido às alterações na configuração familiar e à composição de género da força laboral (Aboim, 2003; Pimenta \& Ribeiro, 2014). O aumento do número de mulheres a trabalhar significa para estas juntar às responsabilidades familiares as responsabilidades de trabalho, enquanto aos homens é exigido maior envolvimento nas tarefas familiares (Allen, Herst, \& Bruck, 2000; Kinnuen \& Mauno, 1998; Perista, 2002; Premeaux, Adkins, \& Mossholder, 2007; Santos \& Gonçalves, 2011).

De facto, a conciliação entre a vida profissional e pessoal tem adquirido especial importância para as instituições, as empresas, as famílias e os próprios indivíduos (Pereira, 2012; Pereira \& Ribeiro, 2014; 
Pichler, 2009), pois cada vez é mais frequente o trabalho interferir na vida familiar e/ou a família influenciar o trabalho (Santos \& Gonçalves, 2011).

Face ao exposto, o presente artigo tem como objetivo apresentar uma revisão histórica do papel do género na conciliação entre o trabalho e a família.

\section{PERSPETIVAS DO GÉNERO NA CONCILIAÇÃO DOS DOMÍNIOS PROFISSIONAL E FAMILIAR}

Perante o papel tradicional de género feminino, as mulheres desempenham a maioria das tarefas familiares, portanto, quanto maior o número de tarefas familiares realizadas por um elemento do casal, maior a probabilidade do papel profissional interferir com o desempenho do papel familiar (Matias, Silva \& Fontaine, 2011; Silva, 2007). Torna-se importante mencionar ainda que o tipo e a qualidade das tarefas familiares desenvolvidas por homens e mulheres são muito díspares. As mulheres são responsáveis pelas tarefas diárias, que implicam mais tempo e estão aliadas ao cuidado dos filhos (Perista, 2002; Torres, 2004) e, por sua vez, as tarefas tradicionalmente masculinas são mais flexíveis em termos de tempo e frequência, e acontecem na sua maioria fora de casa e muitas vezes associada a uma componente lúdica (como, por exemplo, tratar do carro, do jardim, bricolage, etc.). De salientar que muito do trabalho familiar não remunerado, como planear refeições e manter contactos familiares, é subvalorizado e não é ponderado como trabalho, até pelas próprias mulheres (Silva, 2007).

A perspetiva racional explica o efeito do género na perceção da conciliação entre o trabalho e a família, segundo a qual quanto maior é o tempo despendido no domínio profissional, maior é o grau do conflito trabalho-família, e quanto maior o tempo despendido no domínio familiar, maior é a perceção do conflito família-trabalho (Pereira, 2012). Logo, é expectável que ambos os géneros, ao despenderem maior tempo no trabalho remunerado, descrevam maior conflito trabalho-família do que conflito família-trabalho. Podemos igualmente esperar que, por um lado, as mulheres relatem maior conflito família-trabalho do que os homens, visto que são estas que despendem mais tempo em tarefas familiares e domésticas (Hill, 2005; Pereira, 2012; Perista, 2002), e por outro, os homens, uma vez que investem um maior número de horas em trabalho remunerado, percecionem maior conflito trabalho-família (Gutek, Searle \& Klepa, 1991). Contudo, alguns estudos contradizem esta 
perspetiva racional, pois constataram que as mulheres despendem mais tempo no trabalho total (a soma do domínio profissional com o domínio familiar) que os homens, e apesar disso manifestam menor conflito ou níveis idênticos de conflito que o sexo masculino (Grzywacz \& Marks, 2000).

Outra perspetiva é proposta por Gutek e colaboradores (1991), que referem que apesar da família ser mais valorizada que o trabalho, o papel do género determina diferenças entre homens e mulheres. As autoras defendem que o trabalho está associado ao papel de género masculino e a família ao papel de género feminino, e apesar da entrada massiva da mulher no mercado de trabalho, o trabalho doméstico e o cuidado dos filhos continua a ser um papel feminino. Assim, um compromisso semelhante de ambos os géneros nos papéis profissional e familiar poderá originar um resultado díspar em relação ao conflito, visto que o homem foi socializado para priorizar o seu papel profissional e a mulher o seu papel familiar e parental. Para os homens o papel de "ganha-pão" é suficiente para preencher as suas expectativas relacionadas com o seu papel profissional, bem como as relacionadas com o seu papel familiar (Santos, 2008). Deste modo, os homens não percecionam maior sobrecarga ou conflito pois estão a agir de acordo com as expectativas da sociedade. Nas mulheres os papéis profissionais e familiares não apresentam a mesma convergência. O tempo gasto em cada papel preenche somente as expectativas desse papel e não do outro. Sendo esta gestão de distribuição do tempo mais difícil para as mulheres, estas podem percecionar maior conflito trabalho-família (Duxbury \& Higgins, 1991; Pereira 2012). Quando as mulheres cumprem um papel, que não é o que define o género feminino, tendem a compensar realizando um maior número de tarefas familiares. De facto, estudos relatam que as mulheres, em particular as casadas, desempenham um maior número de tarefas familiares (Perista, 2002). Assim, a perceção do conflito trabalho-família poderá ser mais intenso na saúde e no bem-estar das mulheres, enquanto que o impacto família-trabalho poderá ser maior na saúde dos homens (Frone \& Barnes, 1996). O estudo de Duxbury e Higgins (1991) revela que um elevado envolvimento em papéis não tradicionais aumenta o nível de perceção do conflito.

O estudo de Duxbury e Higgins (1991) verificou igualmente que existem diferenças no género no que concerne à perceção da qualidade de vida. Nas mulheres, a qualidade de vida profissional e de vida em geral estão ligadas entre si, nos homens é a qualidade de vida familiar que está 
relacionada com a qualidade de vida geral. Isto porque, as mulheres quando investem nos dois papéis é com o objetivo de obterem uma certa satisfação profissional, e os homens, como as suas tarefas no papel familiar são na sua maioria de natureza recreativa, é expectável que mostrem maior satisfação familiar que as mulheres.

Porém, Frone, Russell e Barnes (1995) referem que não descobriram diferenças no bem-estar de homens e mulheres nas duas direções do conflito, e que os resultados podem ser elucidados com uma abordagem mais focada na teoria da identidade social. O conflito entre o trabalho e a família pode exibir pressão que origine por um lado redução na qualidade de vida num dos domínios, e por outro, uma ameaça na autoimagem do individuo, pois este fica impedido de realizar atividades essenciais que caracterizam a sua identidade. Ao surgir conflito entre o trabalho e a família, este pode interferir na manutenção ou construção da autoimagem profissional e familiar do individuo. Ora, se tanto o domínio profissional como o familiar são importantes, então ambos os géneros desejam ter um elevado desempenho nos dois domínios, e qualquer conflito, independente da sua direção, pode pôr em causa o bem-estar dos indivíduos (Kinnuen \& Mauno,1998).

Constata-se então que segundo o modelo tradicional, para os homens o domínio profissional é tido como central e para as mulheres é o domínio familiar que é definido como central (Duxbury \& Higgins, 1991). Se a mulher está cada vez mais inserida no mercado de trabalho, isto poderá originar a inexistência de alguém para organizar as tarefas domésticas e o cuidado dos filhos (Jacobs \& Gerson, 2004). Se o papel do género feminino está a sofrer alterações, então o papel do género masculino terá que se adaptar a essas alterações, levando a que os homens experimentem cada vez mais tensão entre os dois domínios. De acordo com Kmec (1999), os homens ao constatarem que as necessidades da família não estão a ser garantidas, sentem-se pressionados, apesar de não despenderem tempo extra nas responsabilidades familiares. Os estudos empíricos constatam que o conflito entre o trabalho e a família não é um problema nem exclusivamente masculino, nem exclusivamente feminino. $\mathrm{Na}$ realidade, as atuais estruturas do trabalho e da família dificultam a conciliação das responsabilidades profissionais e familiares para ambos os géneros. 


\section{O PAPEL DO GÉNERO NA CONCILIAÇÃo ENTRE O TRABALHO E A FAMÍLIA}

Um dos acontecimentos mais proeminentes do século XX, foi a inserção das mulheres no mercado de trabalho, o qual se efetuou de uma forma progressiva, massiva e irreversível em toda a estrutura organizacional e em quase todos os ramos de atividade dos países industrializados (Aryee, 1992; Cardoso, 1997; Perista, 2002).

A responsabilidade exclusiva dos homens, até aos anos 50, era o emprego, sendo vistos como o único sustento da família (Duxbury \& Higgins, 1991), enquanto às mulheres cabia cuidar da casa e da família. Além disso, os poucos deveres familiares e domésticos que os homens assumiam eram transferidos para as suas esposas mal surgiam responsabilidades profissionais mais pesadas ou horários mais alargados.

Verifica-se que a partir da década de 60 as mulheres entram mais ativamente no mercado de trabalho, apesar do desenvolvimento industrial nem sempre ter sido acompanhado pelas alterações nas relações sociais. Mas é principalmente nos anos 70 e 80 que surge a feminização no mercado de trabalho e por consequência no domínio público.

Dos anos 50 até à atualidade, a realidade social mudou, o modelo de família tradicional está a tornar-se vestígio de uma sociedade passada. Porém, as mulheres continuam a debater-se com uma posição de desfavorecimento, pois o trabalho é influenciado pelo género dos indivíduos (Santos, 2008; Ferreira, 2010).

Apesar da grande maioria dos trabalhadores ocupar dois ou mais papéis, o nível de envolvimento em cada um deles varia ao longo da vida e de individuo para individuo, entre profissionais de diferentes gerações, diferentes regiões, diferentes profissões, com diferentes estruturas familiares e diferentes modos de vida. Mas para a maioria dos trabalhadores, a família e o trabalho são os dois domínios mais centrais e fundamentais da sua identidade.

As diferenças de género são fundamentais para a compreensão da conciliação entre o trabalho e a família, pois englobam os dois domínios: a relação dos homens com o domínio profissional e a relação das mulheres com o domínio familiar. Embora a participação das mulheres no mercado de trabalho seja cada vez maior, a sociedade ainda perceciona o trabalho doméstico e familiar como sendo tarefas femininas (Aboim, 2003). 
Podemos esperar assim que os homens e as mulheres percecionem a conciliação entre os dois domínios de forma diferenciada e particular. Apesar do evidente papel que o género representa na partilha do trabalho familiar e profissional, verifica-se que poucos estudos se debruçaram na análise dos fatores de género na conciliação entre o trabalho e a família (Parasuraman \& Greenhaus, 2002). A globalidade dos estudos nesta área é somente aplicado em amostras femininas, ou então o papel do género é simplesmente ignorado no estudo (Barnett, 1998). Há, no entanto, algumas exceções, mas cujos resultados são contraditórios: uns reconhecem diferenças significativas na conciliação entre o trabalho e a família, em função do género (Duxbury \& Higgins, 1991; Grzywacz \& Marks, 2000), mas outros estudos não reconhecem tais diferenças (Frone, Russel \& Cooper, 1992b).

Desde o nascimento que tanto o homem como a mulher experimentam diferentes vivências sociais, tornando o papel do investigador mais árduo para desvendar as diferenças de género. Pode-se entender como diferenças de sexo, as diferenças inatas entre homens e mulheres, diferenças devido a variações hormonais, de morfologia ou variações biológicas (Silva, 2007). Logo, as diferenças de género são invariantes ao longo do tempo e através das culturas. No entanto, muitas diferenças estudadas entre homens e mulheres derivam de papéis sociais distintos que uns e outros adotaram ao longo do tempo. A relação por excelência entre a mãe e a criança levou à disparidade dos papéis profissionais e familiares, visto que estabelece que as mães ocupam lugar central na educação dos filhos, ao contrário dos pais cuja função deveria ser especializar-se em domínios alternativos, assumindo um papel instrumental dentro da família (Perista, 2002). Esta disparidade evidente dos papéis para homens e mulheres tem sido examinada por muitos autores, como invariantes ao longo do tempo e consequentemente como diferenças de sexo e não de género. As diferenças de género compreendem as diferentes experiências sociais e as expectativas do individuo perante os papéis e atitudes de género, logo, o género é essencialmente o resultado dos processos sociais e culturais. A confusão surge quando as diferenças entre homens e mulheres, diferenças que retratam uma cultura e um período de tempo próprio, são abordadas como se tratasse de diferenças de sexo (Silva, 2007).

Nas ciências sociais nos anos 50, a integração do género ao sexo era adotada, pois homens e mulheres operavam em mundos segregados (Pinto, 2002). Apesar destes ideais sociais serem validados apenas 
naquele tempo, levaram a normas e preceitos que ainda hoje influenciam a definição dos papéis familiares e profissionais. Como era baixo o acumular de papéis sociais das mulheres e dos homens, a disparidade entre os efeitos do sexo e os efeitos destes papéis sociais é complexificada (Silva, 2007).

Apenas quando os homens e as mulheres possuírem experiências idênticas, é que se poderá procurar observar as diferenças de género. A entrada gradual das mulheres casadas no mercado de trabalho e o modelo de mãe trabalhadora tornou pertinente a relação entre o trabalho e o stress psicológico. Visto que o papel de trabalhadora foi adicionado aos outros papéis femininos, supõe-se que as mulheres se encontrem sob maior stress e pressão que os homens (Duxbury \& Higgins, 1991; Hill, 2005; Pinto, 2002).

As investigações sobre a conciliação entre o trabalho e a família têm focado o seu estudo na prática da conciliação das mulheres, deixando para segundo plano o estudo da prática dos homens (Hill, 2005). Perante tal cenário, assume-se que as mulheres percecionam o conflito, entre os domínios profissional e familiar, mais intensamente. Segundo Perista (2002), as mulheres ainda reúnem um maior número de responsabilidades familiares e domésticas do que os homens, além disso ainda dispõem de empregos de menor estatuto com menos benefícios para a harmonização destes dois domínios. Assim, as mulheres teriam maiores probabilidades de experienciar o conflito e menores probabilidades para experienciar o enriquecimento (Grzywacz \& Bass, 2003). No entanto, Baruch, Biener e Barnett (1986) referem que as mulheres que desempenham diversos papéis sentem-se menos deprimidas e percecionam uma maior autoestima. Todavia, a qualidade dos papéis desempenhados é fundamental para o aumento da autoestima (Pinto, 2002). Assim, reconhecer que os homens que desempenham um papel profissional poderão vivenciar os mesmos desafios e que estas questões são igualmente pertinentes para eles, são observações mais recentes, apesar de já suportadas em resultados empíricos (Hill, 2005).

Verifica-se uma disparidade de resultados no estudo das diferenças de género. Se, por um lado certos autores revelam que as mulheres vivenciam mais stress e tensão ao participarem em vários domínios e deste modo percecionam maior conflito que os homens (Hill, 2005; Marshall \& Barnett, 1993). Por outro, existem estudos que demonstram que as mulheres têm maior capacidade de gerir o conflito entre os dois domínios (Kirchmeyer, 1992a,b; 1993). Há ainda autores que verificam 
diferenças de género mas apenas num sentido, isto é, as mulheres percecionam maior conflito trabalho-família que os homens (Gutek et al., 1991; Kmec, 1999; Pereira, 2012), e outros estudos ainda demonstram uma ausência de diferenças de género, qualquer que seja o sentido (Byron, 2005; Frone, Russell \& Cooper, 1992a; Kinnunen \& Mauno, 1998).

Em relação à perceção do enriquecimento, mantêm-se os resultados díspares, enquanto certos estudos relatam que as mulheres percecionam maior enriquecimento que os homens (Aryee, Srinivas, \& Tan 2005; Pereira, 2012), sobretudo no sentido trabalho-família (Grzywacz et al., 2002; Grzywacz \& Marks, 2000; Marshall \& Barnett, 1993), outros estudos não evidenciam quaisquer diferenças entre os dois géneros (Hill, 2005; Kirchmeyer, 1992b).

Pela revisão de literatura sobre o género e a conciliação de responsabilidades familiares e profissionais, verifica-se que a análise é fundamentalmente sobre as diferenças na perceção do conflito entre o trabalho e a família, descurando as diferenças na análise do enriquecimento entre o trabalho e a família.

\section{REFLEXÕES CONCLUSIVAS}

O número crescente de famílias de duplo-rendimento originou diversas alterações nos modelos de compatibilização entre o domínio profissional e familiar (Santos, 2008).

Sem dúvida, que a divisão tradicional do trabalho entre homens e mulheres, onde o trabalho remunerado está relacionado ao homem e o não remunerado à mulher, não é atualmente uma opção consensual. Os tradicionais papéis de género sofrem alterações com a evolução da sociedade, e as mudanças sociais que temos vindo a assistir têm implicações evidentes tanto nos homens como nas mulheres (Aboim, 2003). Por um lado, às mulheres é cada vez mais solicitado um ritmo de trabalho exigente e intenso, reduzindo a sua disponibilidade para as tarefas familiares, e por outro, o envolvimento crescente dos homens na esfera familiar implica uma mudança nas suas prioridades. Como se constatou, as investigações são influenciadas por valores sociais, e a maior parte dos estudos direcionam o seu foco sobre o género feminino, ignorando os conflitos que o género masculino tem de gerir (Barnett, 1998). Isto limita a procura de soluções de igual partilha de responsabilidades entre homens e mulheres. As mulheres, não raras 
vezes, têm que optar entre o seu projeto de vida familiar e o seu projeto profissional. Por vezes, os ajustes de curto prazo que algumas mulheres fazem acabam por se repercutir a longo prazo. Se uma mulher optar por adequar as exigências profissionais para cumprir as necessidades familiares, ou então evitar essas necessidades não tendo filhos ou adiando a maternidade, ambas as opções são limitativas (Torres, 2004). Igualmente, os pais são compelidos pela sociedade e pela cultura organizacional, o que os impede de investir menos tempo no papel profissional em prol do cuidado dos filhos ou de uma maior partilha das responsabilidades domésticas.

Perante os inconstantes resultados do papel do género, uma análise do conflito entre o trabalho e a família e do enriquecimento entre o trabalho e a família distinguida por género, possibilitará direcionar uma intervenção mais adequada e ajustada às necessidades e vivências de homens e mulheres.

\section{BIBLIOGRAFIA}

Aboim, S. (2003). Evolução das estruturas domésticas. Sociologia, Problemas e Práticas, 43, 13-30.

Allen, T. D., Herst, D. E., \& Bruck, C. S. (2000). Consequences associated with work to- family conflict: a review and agenda for future research. Journal of Occupational Health Psychology, 5, 2, 278-308.

Aryee, S. (1992). Antecedents and outcomes of work-family conflict among married professional women: Evidence from Singapore. Human Relations, 45 (8), 813-837.

Aryee, S, Srinivas, E., \& Tan, H. (2005). Rhythms of life: antecedents and outcomes of work-family balance in employed parents. Journal of Applied Psychology, 90 (1), 132-146.

Barnett, R. (1998). Toward a review and reconceptualization of the work/family literature. Genetic, Social \& General Psychology Monographs, 124 (2), 125-153.

Baruch, G., Biener, L., \& Barnett, R. (1986). Women and gender in research on work and family stress. American Psychologist, 42 (2), 130-136.

Byron, K. (2005). A meta-analytic review of work-family conflict and its antecedents. Journal of Vocational Behavior, 67, 169-198. 
Cardoso, A. (1997). Trabalho feminino em Portugal: Valorização da mulher na economia ou valorização da economia com a mulher? Lisboa: CITE.

Duxbury, L.E., \& Higgins, L.A. (1991). Gender differences in work/family conflict. Journal of Applied Psychology, 78 (1), 60-73.

Ferreira, V. (2010). A evolução das desigualdades entre salários masculinos e femininos: um percurso irregular. In V. Ferreira (Org.), A igualdade de mulheres e homens no trabalho e no emprego em Portugal: Políticas e Circunstâncias (pp. 139-190). Lisboa: Comissão para a Igualdade no Trabalho e no Emprego.

Frone, M. R., \& Barnes, G. M. (1996). Work-Family Conflict, gender and health related outcomes: a study of employed parents in two community samples. Journal of Occupational Health Psychology, 1, 57, 57-69.

Frone, M., Russell, M., \& Cooper, M. (1992a). Antecedents and outcomes of work-family conflict: testing a model of the work-family interface. Journal of Applied Psychology, 77 (1), 65-78.

Frone, M., Russell, M., \& Cooper, M. (1992b). Prevalence of workfamily conflict: are work and family boundaries asymmetrically permeable? Journal of Organizational Behavior, 13, 723-729.

Frone, M., Russell, M., \& Barnes, G. (1995). Job stressors, job involvement and employee health: a test of identity theory. Journal of Occupational and Organizational Psychology, 68, 1-11.

Grzywacz, J., \& Bass, B. (2003). Work, family and mental health: testing different models of work/family fit. Journal of Marriage and Family, 65, 248-262.

Grzywacz, J. G., \& Marks, N.F. (2000). Reconceptualising the workfamily interface: An ecological perspective on the correlates of positive and negative spillover between work and family. Journal of Occupational Health Psychology, 5, 111-126.

Gutek, B., Searle, S., \& Klepa, L. (1991). Rational Versus Gender Role Explanations for Work-Family Conflict. Journal of Applied Psychology, 76 (4), 560-568.

Hill, J. (2005). Work-Family facilitation and conflict, working fathers and mothers, work-family stressors and support. Journal of Family Issues, 26 (6), 793-819.

Jacobs, J. A., \& Gerson, K. (2004). The time divide: work, family and gender inequality. Harvard University Press: Cambridge. 
Kinnuen, U., \& Mauno, S. (1998). Antecedents and outcomes of workfamily conflict and its relationship with satisfaction and well-being: a one year longitudinal study on gender difference. Work \& Stress, 18 (1), 157-177.

Kirchmeyer, C. (1992a). Nonwork participation and work attitudes: a test of scarcity vs. expansion models of person resources. Human Relations, 45 (8), 775-795.

Kirchmeyer, C. (1992b). Perceptions of nonwork-to-work spillover: challenging the common view of conflict-ridden domain relationships. Basic and Applied Psychology, 13 (2), 231-249.

Kmec, J. (1999). Multiple aspects of work-family conflict. Sociological Focus, 32 (3), 265-285.

Matias, M., Silva, A., \& Fontaine, A. M. (2011). Conciliação de papéis e parentalidade: efeitos de género e estatuto parental. Exedra, 5, 57-76.

Marshall, N., \& Barnett, R. (1993). Work-family strains and gains among two-earner couples. Journal of Community Psychology, 21, 64-78.

Parasuraman, S., \& Greenhaus, J. (2002). Toward reducing some critical gaps in work-family research. Human Resource Management Review, 12, 299-312.

Pereira, S. (2012). O equilíbrio trabalho/família nos advogados: Diferenças de género. Dissertação de Mestrado em Gestão: Especialização em Gestão de Recursos Humanos. Centro regional de Viseu da Universidade Católica Portuguesa. Viseu.

Pereira, S., \& Ribeiro, C. (2014). O equilíbrio trabalho/família: revisão de literatura. Gestão e Desenvolvimento, 22, 73-88.

Perista, H. (2002). Género e trabalho não pago: os tempos das mulheres e os tempos dos homens. Análise Social, 37 (163), 447-474.

Pimenta, S., \& Ribeiro, M. T. (2014). Trabalho-Família: Uma questão de equilíbrio? In M. J. Chambel \& M. T. R. Ribeiro (Coord.), A relação entre o trabalho e a família (pp. 12-40). Lisboa: Editora RH.

Pimenta, S., \& Ribeiro, M. T. (2014). O equilíbrio trabalho-família e suas repercussões no bem-estar. In M. J. Chambel \& M. T. R. Ribeiro (Coord.), A relação entre o trabalho e a família (pp. 82-116). Lisboa: Editora RH.

Pichler, F. (2009). Determinants of Work-life balance: shortcomings in the contemporary measurement of WLB in large-scale survey. Soc Indic Research, 92, 449-469. 
Pinto, A. (2002). As diferenças de género na perceção do conflito trabalho-família. Dissertação de Mestrado. Universidade do Minho. Braga.

Premeaux, S. F., Adkins, C. L., \& Mossholder, K. M. (2007). Balancing work and family: a field study of multi-dimensional, multi-role workfamily conflict. Journal of Organizational Behavior, 28, 705-727.

Santos, G. (2008). Género, carreiras e a relação entre o trabalho e a família: uma perspetiva de gestão. Acedido a 12 de Abril de $2012 \mathrm{em}$ http://pais-para-sempre.blogspot.pt/2008/10/gnero-carreiras-e-relaoentre-o.html

Santos, J., \& Gonçalves, G. (2011). Contribuição para a adaptação portuguesa da escala de conflito trabalho-família e família-trabalho de Netmeyer, Boles e McMurrian (artigo não publicado).

Silva, M. (2007). Vida Profissional e Familiar: Padrões de Conflito e Facilitação na Gestão de Múltiplos Papéis. Dissertação de Mestrado em Psicologia. F.P.C.E.U.P. Porto.

Torres, A. (2004). Vida conjugal e trabalho: uma perspetiva sociológica. Oeiras: Celta Editora. 